\title{
Investigation of smooth wave fronts using SLM-based phase retrieval and a phase diffuser
}

\author{
M. Agour \\ agour@bias.de \\ P. F. Almoro \\ C. Falldorf
}

\author{
Bremer Institut für angewandte Strahltechnik, Klagenfurter Strasse 2, 28359 Bremen, Germany \\ Physics Department, Faculty of Science, Aswan University, 81528 Aswan, Egypt \\ National Institute of Physics, University of the Philippines, Diliman, Quezon City, 1101 Philippines
}

Bremer Institut für angewandte Strahltechnik, Klagenfurter Strasse 2, 28359 Bremen, Germany

\begin{abstract}
A phase retrieval technique using a spatial light modulator (SLM) and a phase diffuser for a fast reconstruction of smooth wave fronts is demonstrated experimentally. Diffuse illumination of a smooth test object with the aid of a phase diffuser (maximum phase shift, $\Delta \phi=0.85 \pi)$ results in a significant diversity in the intensity measurements which, in turn, is beneficial for a non-stagnating iterative phase reconstruction. The use of the SLM enables accurate and fast speckle intensity recording and active correction of misalignments in the setup. The effectiveness of the technique is demonstrated in the optical testing of lenses.
\end{abstract}

[DOI: http://dx.doi.org/10.2971/jeos.2012.12046]

Keywords: Phase retrieval, wave front sensing, rough surfaces, spatial light modulator

\section{INTRODUCTION}

Phase retrieval is an approach to reconstruct the phase distribution of an optical wave field from a set of recorded intensity patterns without the requirement of a distinct reference wave. Recovering the phase from intensity measurements can be described as an inverse problem which is mostly solved iteratively. An important requirement to successful phase retrieval is diversity or local differences in the captured set of intensity patterns [1]. From earlier techniques that use two intensities [2], recent trends favour the use of multiple intensities [3]. To generate the multiple intensities, recording modalities include changing the light source wavelength [4], physical translation of an aperture [5] or a specimen [6] along the transverse direction and physical displacement of the recording camera along the axial direction [7]. For the reconstruction [7], an iterative algorithm based on the generalized projections is used to recover the phase of the investigated wave field. The algorithm starts with a guess of the complex amplitude of the wave field at the first plane. This guess wave field consists of a random phase and real amplitude calculated from the square root of the measured intensity across that plane. Using the plane wave decomposition approach [8], the wave field formed is propagated numerically to the adjacent measurement plane. The process of combining the calculated phase and the real amplitude followed by the wave propagation is repeated until the last measurement plane is reached. Thereafter, the propagation proceeds from last plane to first plane. The sequential process is repeated until no significant difference between two phase distributions obtained in two consecutive iterations is observed. The benefit of using multiple intensities is the enforcement of the associated inverse problem resulting in the faster convergence compared to the techniques that use only two intensities [7]. The technique has been demonstrated on rough test objects cite7. A major drawback of this technique is the long measurement time due to the mechanical adjustment of the recording planes [7]. In addition, the technique has limited applications in cases of smooth test objects. Because smooth wave fronts do not vary significantly over a short axial distance, this results in a lack of diversity between the captured intensity patterns. Compounded by inadequate sampling due to detectors quantization, this lack of diversity leads to the stagnation problem in the phase calculation. In order to adapt the multiple-intensity phase retrieval technique to smooth wave fronts, the use of diffuse illumination with a partially-developed speckle field has been shown to enhance the quality of reconstructions [9]. The resulting set of high contrast speckle intensity patterns makes the technique robust against quantization errors [10]. The diffuse illumination phase retrieval technique has been demonstrated in the reconstruction of wave fronts from smooth transmitting samples [9] and specularly reflecting objects [11]. The mechanical movement of the camera across the multiple planes, however, makes the technique slow, tedious and prone to misalignments. In this study, we are interested in another modality for generating the multiple intensity patterns in which the propagation planes are accessed virtually using an active Opto-electronic device.

In order to alleviate the issues related to the mechanical movement of the camera, a technique based on the use of a spatial light modulator (SLM) has recently been introduced $[12,13]$. Instead of the axial displacement of the recording camera [7], the SLM facilitates for a virtual propagation of 
the wave field, thus, allowing fast and accurate single-plane intensity recording $[12,13]$. This technique is referred to as SLM-based phase retrieval (SLM-PR) technique and a schematic of its basic setup is shown by Figure 1. The SLMPR works by considering the propagation of a wave field between two parallel planes as a linear invariant system with the transfer function of free space propagation. Due to the fact that the transfer function of free space propagation is a pure phasor, the whole propagation model can be realized by means of a phase-only SLM placed at the Fourier domain of a $4 f$-imaging system. Within the SLM-PR technique, the SLM modifies electronically the wave field by means of the transfer function of free space propagation, which is a pure phase function. Accordingly, a set of intensity measurements associated to different propagated states can be captured across a single camera plane [12]. In addition to the enhanced speed and accuracy of the intensity recording, the SLM-PR setup allows active correction for any misalignments in the setup. The effectiveness of the SLM-PR was demonstrated in the optical inspection of technical components under thermal loading [14, 15].

In this work, we demonstrate experimentally a novel technique for fast and accurate reconstruction of smooth test wave fronts using the SLM-PR with diffuse illumination. Upon modulations from a diffuser phase plate and the SLM, the transmitted object wave intensity exhibits the desired diversity. Hence, the main advantage of the new SLM-PR technique is the fast and accurate speckle recording paving the way for enhanced reconstruction of smooth wave fronts. As experimental demonstration, the technique is applied in the shape measurement of thin lenses.

\section{PRINCIPLES OF THE PROPOSED TECHNIQUE}

The design parameters of the setup, complex transmittance displayed on the SLM and the iterative reconstruction algorithm will be reviewed. The physical features of the diffuser phase plate will be discussed in the context of the phase shift introduced to incident light and the formation of a speckle field.

\subsection{Design parameters of the SLM-PR setup}

Here we give the design parameters of the SLM-PR setup presented in Figure 1. As an illumination beam, a plane wave generated by a frequency doubled Nd:YAG with a wavelength of $\lambda=532 \mathrm{~nm}$ is used. The SLM used is a reflective liquid crystal phase-only SLM supplied by Holoeye Photonics AG [17]. It has a pixel pitch of $\Delta p=8 \mu \mathrm{m}$ and is configured to provide full $2 \pi$ phase modulation with linear electro-optical characteristics. The camera used is a F-505b/Pike from Allied Vision Technologies $\mathrm{GmbH}$ that has 2452 × 2054 pixels with a pixel pitch of $3.45 \times 3.45 \mu \mathrm{m}$.

An important consideration in the design of the SLM-PR setup is the minimization of the disturbing effects of the higher diffraction orders, i.e., a set of laterally shifted replicas of the

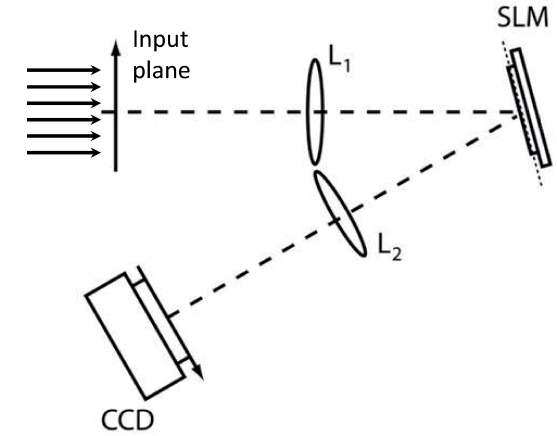

FIG. 1 Sketch of the SLM-PR setup. SLM refers to a phase-only spatial light modulator, CCD or charge-coupled device is the recording camera and $L_{1}$ and $L_{2}$ are identical lenses.

wave field, arising from the discrete nature of the SLM sensor [18]. To avoid the overlapping of the diffraction orders, the focal length of the lenses is selected to be $f=150 \mathrm{~mm}$. In this case, the distance between the diffraction orders is 10 $\mathrm{mm}$ in any direction. Based on the camera resolution and pixel pitch, the distance between the diffraction orders is made significantly larger than the dimension of the camera sensor $(8.5 \mathrm{~mm} \times 7.0 \mathrm{~mm})$. Subsequently, the constructed setup does not suffer from systematic distortions caused by the SLM. More details on selecting parameters to avoid the overlapping of the diffraction orders in the Fourier domain can be found in Ref. [19]. Furthermore, the structure of the wave field in the sensor domain and its dependence on the discrete nature of the SLM has been studied in Ref. [20].

\subsection{Controlling the complex transmittance to be generated by the SLM}

The propagation scheme between two axially-displaced planes can be realized by means of an optical linear filtering processor using SLM as mentioned in the introduction section. As depicted in Figure 1, the filtering setup is based on a $4 f$-configuration with the SLM as an active element located in the Fourier domain.

With the following pure phase transfer function for free space propagation being displayed by the SLM, an output wave field can be obtained across the CCD plane based on a propagated representation of the wave field at the input plane [8]:

$$
\boldsymbol{H}_{z}(\xi)=\exp \left[i \frac{2 \pi z}{\lambda} \sqrt{1-\lambda^{2}|\xi|^{2}}\right]
$$

where $z$ denotes the distance between the two planes and the vector $\xi=\left(\xi_{i}, \xi_{j}\right)$ represents a vector of the frequencies in two dimensional space. To control the complex transmittance generated by the SLM, it is necessary to consider the Fourier transform property of the lenses. Consequently, the transfer function for free space propagation given by Eq. 1 has to be rewritten using the substitution:

$$
\xi=v \cdot(\lambda f)^{-1}
$$

This relation gives the correlation of the frequency domain $\xi$ and the spatial domain $v$ in the back focal plane of the first lens. Accordingly, Eq. 1 can be brought into its spatial repre- 
sentation form [13]:

$$
H_{z}(v)=\exp \left[i \frac{2 \pi z}{\lambda} \sqrt{1-\frac{1}{f^{2}}|v|^{2}}\right]
$$

Eq. 3 represents a continuous representation of the transfer function for free space propagation. Due to the fact that the SLM is a device that has discrete structures, a discrete representation of the transfer function is needed. According to the sampling theory, the term $H_{z}(v)$ may be modelled using the following relation:

$$
\boldsymbol{H}_{z}[m, n]=\exp \left[i \frac{2 \pi z}{\lambda} \sqrt{1-\frac{\Delta p^{2}}{f^{2}}\left(m^{2}+n^{2}\right)}\right]
$$

Here, $H_{z}[m, n]$ denotes a discrete distribution of the transfer function which consists of $m$ and $n$ pixels and $\Delta p$ is the pixel pitch of the SLM. Eq. 4 gives the real distribution of the transfer function which then is displayed by the SLM. Substituting the design parameters given in the preceding subsection, a set of intensity measurements associated with multiple propagation states of the initial wave field can be realized across the sensor plane by choosing the propagation distance $z$ accordingly.

\subsection{Properties of the fabricated diffuser phase plate}

The main purpose of using a random phase diffuser (RPD) is to introduce a controlled randomization on an incident plane wave resulting in a significant diversity in both the amplitude and phase. Compared to using an ordinary ground glass diffuser plate that results in a complete randomization of light, the use of RPD having certain features provides a mechanism to achieve enhanced axial intensity variation. Compared to amplitude-type diffusers, a phase-type diffuser is energy efficient [21].

Based on Huygen's principle, every point on an illuminated rough surface becomes a source of spherical wavelets. Subsequently, for the case of rough surfaces, the superposition of such spherical wavelets across a sufficiently distant observation plane generates a speckle field [22]. The type of the generated speckle field depends on the roughness of the diffusing plate and the $\lambda$ of the coherent light. If the surface is optically rough, i.e., the surface indentations are much bigger than $\lambda$, a fully-developed objective speckle field is generated [23]. Using a fully-developed speckle field with a fully randomized phase distribution does not lend itself useful for the reconstruction of the incident smooth wave front [21]. Therefore, we would like to fabricate an RPD which generates a partially-developed speckle field when illuminated with a coherent light of specific $\lambda$. A partially-developed speckle field can be achieved if the roughness height of the fabricated plate will result in a maximum phase shift that is less than $\pi$. In this case, part of the light incident on such surface will be scattered and another part will be unperturbed i.e., a transmitted light as if there is no RPD. The superposition of these two parts generates the partially-developed speckle field.

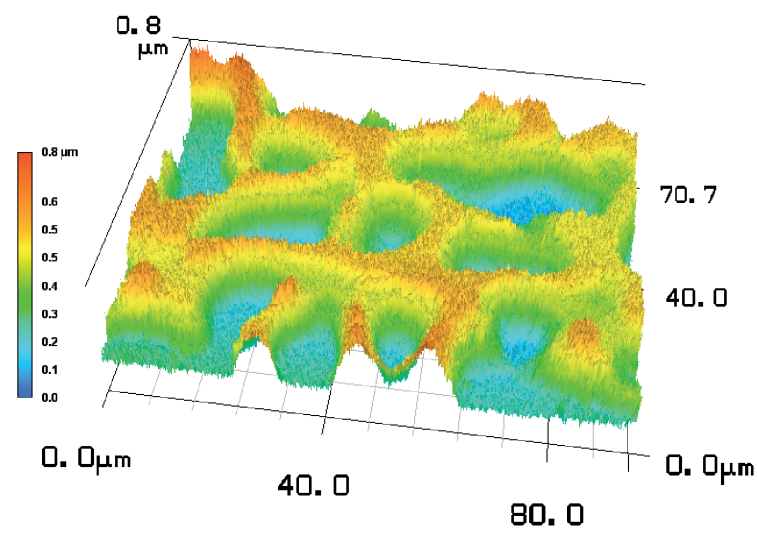

FIG. 2 Three-dimensional height map of the fabricated RPD plate for a cross-section of $95 \times 65 \mu \mathrm{m}^{2}$ measured by Keyence microscope.

Here we briefly summarize the photolithographic process employed in the fabrication of the RPD. A more detailed discussion of the process can be found in Ref. [21]. The RPD plate was fabricated using ultraviolet (UV) photolithography on a photoresist plate (S1805). For this purpose a collimated helium-cadmium laser, with $\lambda=442 \mathrm{~nm}$ was incident on a ground glass (diffuser) forming a fully-developed speckle beam. The speckle field was used to illuminate the photoresist plate (photoresist thickness: $500 \mathrm{~nm}$; refractive index: 1.65). In order to fabricate the RPD with the desired scattering properties, the mean speckle size and maximum depth of the indentations are important. The mean speckle size was controlled by adjusting the distance between the diffuser and the photoresist plate, the diameter of the diffuser which is controlled by a diaphragm and $\lambda$. The maximum depth of the indentations, on the other hand, depends on the UV exposure (intensity and duration) and the chemical development of the photoresist.

For the application of the fabricated RPD in diffused illumination SLM-PR, it is imperative that the particular RPD element to be used is tuned to the wavelength of the light source used (i.e., it will result in maximum phase shift less than $\pi$ at a particular wavelength).

We use 3D Laser Scanning Microscope (Keyence) to investigate the structures of the fabricated RPD plate. Figure 2 shows the $3 \mathrm{D}$ height map of the fabricated RPD that has a surface shape profile likened to islands and trenches.

The maximum roughness height $\mathrm{h}$ (or depth of the indentations) of the RPD plate is $0.348 \mu \mathrm{m}$. Substituting the parameters of the fabricated RPD, the maximum phase shift $\Delta \phi$ given by

$$
\Delta \phi=\frac{2 \pi}{\lambda} \cdot\left(n_{\text {resist }}-n_{\text {air }}\right) \cdot h,
$$

is found to be $0.85 \pi$.

The mean roughness size along the transverse directions (average of the widths of the islands and trenches on the RPD) is $22 \mu \mathrm{m}$. When the fabricated RPD plate is illuminated with 


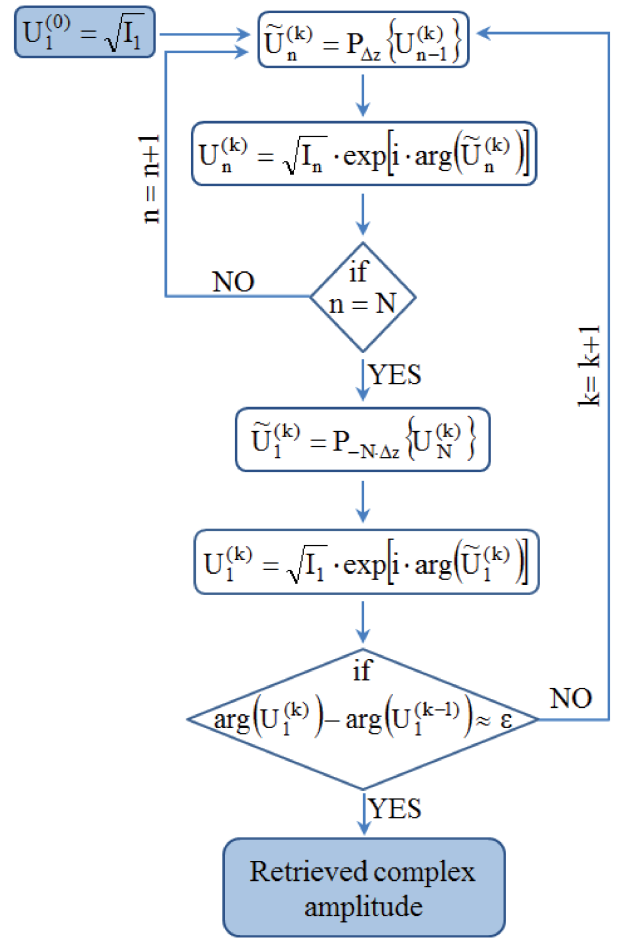

FIC. 3 Flowchart of the iterative phase retrieval, where $\mathrm{n}$ represents the propagation plane number and it varies from 1 to $\mathrm{N}, \mathrm{k}$ stands for the iteration number and $\tilde{\mathrm{U}}$ and $\mathrm{U}$ denote the resulting complex amplitudes directly after applying the propagation operator and after exchanging the real amplitude with the square root of the measured intensity.

a coherent light beam, having a beam diameter $(10 \mathrm{~cm})$ much greater than the mean roughness size along the transverse directions, a partially-developed speckle field is generated.

\subsection{Description of the phase retrieval process}

The iterative phase retrieval algorithm is based on the reconstruction approach that was developed in Ref. [7]. To recover the phase, a set of intensity patterns $\{1, \ldots, N\}$ of the scattered light across a sequence of $N$ propagation planes is used. Figure 3 shows a flowchart of the phase retrieval algorithm.

The algorithm starts from an initial propagation plane, $\{1\}$, where the wave field amplitude is obtained from the square root of the corresponding intensity pattern. Because the initial phase distribution is unknown, here it was assumed to be zero. Then, the complex amplitude $U_{1}$ was propagated numerically to the adjacent plane, $\{2\}$, using the plane wave decomposition approach [8]:

$$
P_{\Delta z}\left\{U_{2}\right\}=F^{-1}\left\{F\left\{U_{1}\right\} \cdot H_{z}\right\}
$$

Here, $F\{\}$ and $F^{-1}\{\}$ denote the 2D Fourier transform and its inversion, respectively, and $H_{z}$ is the transfer function for free space propagation defined by Eq. 1. It is remarked that the calculation of the wave field with amplitude based on the measured intensity pattern is the only constraint imposed in the algorithm.

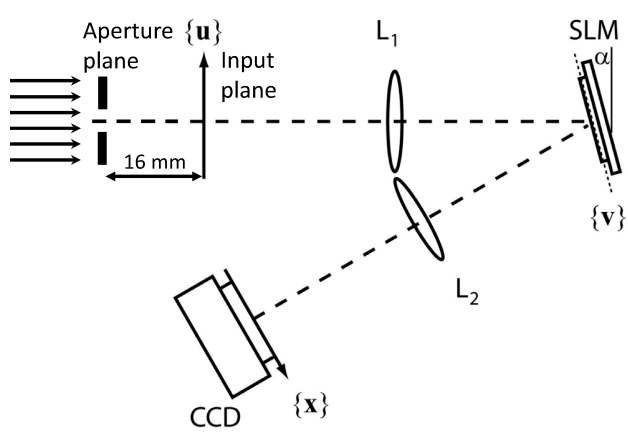

FIG. 4 A plane wave is incident on an aperture (without diffuser phase plate) and the transmitted light is directed to SLM-PR setup. $\alpha$ is a small tilt angle, $u, x$ and $v$ are the coordinates at the input, the camera and the common Fourier planes, respectively. $L_{1}$ and $L_{2}$ denote two identical lenses and SLM refers to the spatial light modulator.

The process of calculating the wave field followed by wave propagation is repeated until the last plane, $\{N\}$, is reached. The process then proceeds directly from last plane to the first plane. The whole procedure is repeated, while the change of phase retrieved at the first plane in two successive iterations is calculated in each cycle. The algorithm converges to the iterative solution, if there is no significant change in the phase is observed.

\section{EXPERIMENTAL RESULTS}

\subsection{Experimental control for proof of principle demonstration}

In the following we demonstrate the benefit of using the setup with diffuser in the reconstructions of simple smooth wave fronts by comparing it to the case when no diffuser is used. The complex amplitudes of a plane wave for two cases: 1) incident along, and, 2) incident at an angle with the optical axis, are evaluated using the SLM-PR setup with and without a diffuser.

In the first case, a plane wave parallel to the optical axis is incident on an aperture having a diameter of $2.5 \mathrm{~mm}$ and placed at $16 \mathrm{~mm}$ away from the input plane (front focal plane of the first lens). The wave field is then directed to the SLM-PR setup, as shown by Figure 4.

In order to recover the phase distribution across the input plane, a sequence of 10 intensity distributions of the light scattered by the aperture was captured by means of a CCD array in the sensor domain. The intensity distributions are associated with the different propagation states corresponding to a set of recording planes that are separated by $\Delta z=2 \mathrm{~mm}$ along the optical axis. The corresponding transfer functions for free space propagation were generated by means of the SLM in the Fourier domain according to Eq. 3. Using the SLM, no mechanical adjustment is required throughout the recording process. Additionally, recording the whole set of intensities took less than a second because the switching time of the SLM used is about $50 \mathrm{~ms}$. An example of the 

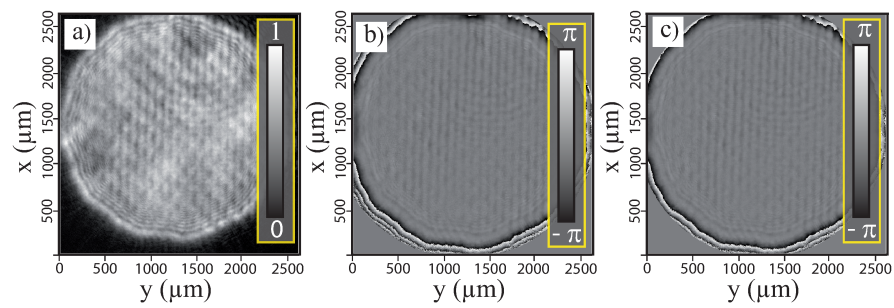

FIG. 5 Experimental results for setup without phase diffuser: (a) Observed real amplitude in the sensor domain. Phase recovered after 200 iterations for a plane wave that is incident (b) parallel to and (c) at an angle with respect to the optical axis of the setup. (All distributions: $750 \times 750$ pixels with a pixel pitch of $3.45 \mu \mathrm{m}$ ).

captured intensity at the imaging plane is shown by Figure 5a.

In the second case, the plane wave was incident at a controlled tilt angle of 0.085 degrees with respect to the optical axis. In this case, it is noted that the impulse response spot generated across the Fourier domain is shifted compared to the one resulting from a plane wave incident parallel to the optical axis of the setup. Consequently, the complex amplitude generated across the CCD plane is shifted and modulated with a linear phase ramp in accordance with our previous modelling of the SLM-PR setup, (see Refs. $[13,16]$ ). Using the alignment method presented in Ref. [13] the SLM-PR setup was aligned. It is remarked that the alignment approach eliminates only the shift effect occurred at the CCD plane and the ramp modulation still remains.

The captured intensities in both cases were subjected to the iterative scheme to retrieve the phase distributions. The phase recovered across the input plane after 200 iterations is shown by Figure 5(b) and 5(c). The recovered phase distributions in both cases, especially in the central portion of the aperture area, are the same. This means that at least one of them is not physically correct, because the linear phase ramp is not retrieved in the case of the tilted plane wave. This also means that the iterative approach converges to a wrong solution which is due to the lack of significant diversity in the captured set of intensity measurements. It is noted that the observed circular fringes retrieved near the aperture rim are attributed to the diversity introduced by the diffraction of the plane wave by the space-limited aperture which is used to adapt the size of speckle across the camera plane.

Let us now introduce the desired diversity by inserting the RPD directly in front of the aperture plane and then illuminate the input plane with the resulting partially-developed speckle field. The distance from RPD plane and the input plane is $18 \mathrm{~mm}$. Two sets, each of 10 intensity measurements, are captured for a plane wave that is incident parallel to and at an angle with respect to the optical axis of the setup. One example of the captured intensity at the imaging plane is shown by Figure 6(a). Figures 6(b) and 6(c) show the phases recovered across the input plane for 200 iterations when the plane wave is incident parallel to and at the tilt angle of 0.085 degrees with respect to the optical axis, respectively.
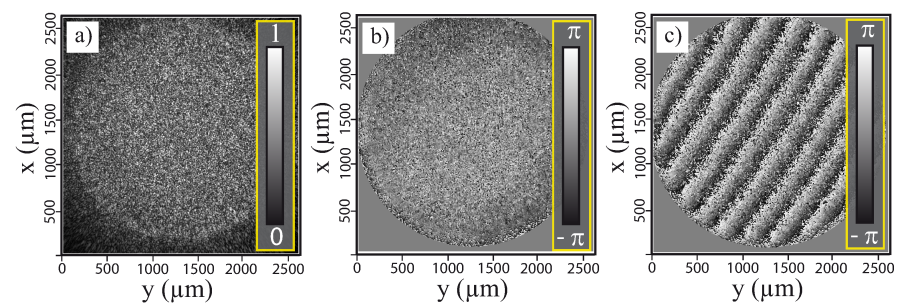

FIG. 6 Experimental results obtained after introducing the desired diversity by means of the fabricated RPD plate: (a) Observed real amplitude in the sensor domain. Phase recovered after 200 iterations for a plane wave that is incident parallel to (b) and, at an angle with respect to the optical axis of the setup (c). (All distributions: $750 \times 750$ pixels with a pixel pitch of $3.45 \mu \mathrm{m}$ ).

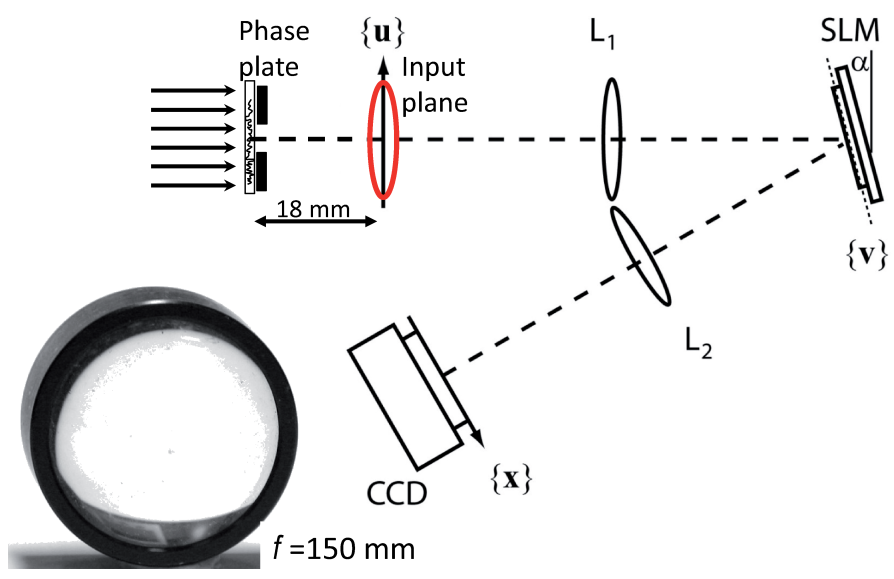

FIG. 7 The scheme of the SLM-PR setup used to determine the phase variation of a plane wave after its modulation with a thin lens having a focal length of $f=150$ $\mathrm{mm}$.

The phase distribution of the plane wave arriving parallel to the input plane (Figure 6(b) has a constant phase value. While the phase distribution of the plane wave arriving at an angle at the input plane (Figure 6(c) has diagonal fringes indicating the tilt. The ability to distinguish the two cases of the plane wave incidence demonstrates successful phase retrieval. Based on the 9 fringes across the aperture diameter, the tilt angle is found to be 0.00158 rad which corresponds approximately to $0.09^{\circ}$.

Comparing this value and the angle of the incident plane wave proves that the retrieved phase of the tilted plane wave is correct. Please note that, the observed high frequency phase variation is expected and is due to the scattered wave component of the partially-developed speckle field.

\subsection{Investigation of smooth wave fronts}

Here, the generated speckle field is then used to illuminate a centred thin lens having a focal length of $f=150 \mathrm{~mm}$ and positioned at the object input plane of the setup, as shown by Figure 7. The inset in Figure 7 shows also a photo of the test object used.

The diffuser is $18 \mathrm{~mm}$ away from the object plane to adequately sample the generated speckle according to the pixel pitch of the CCD. A sequence of 10 spatially separated recording planes is selected using the SLM-PR setup, where the 


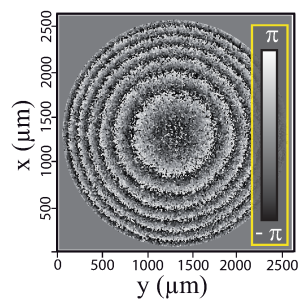

FIG. 8 Retrieved phase of the investigated lens across the object plane after 200 iterations.
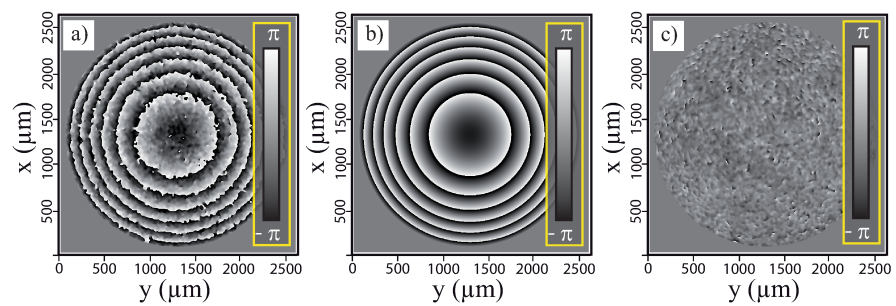

FIG. 9 Comparison between the phase distributions of the test lens obtained from the experiment and simulation: (a) Filtered version of the retrieved phase distribution of the thin lens shown by Figure 8. (b) Numerical simulation of the test lens refractive profile. (c) Subtraction of the two phase distributions.

distance between successive planes is $2 \mathrm{~mm}$. Subsequently, the speckle measurements with sufficient intensity variation are subjected to the iterative algorithm discussed in Section 2.4. Figure 8 shows a representative phase map of the smooth test wave front using the proposed technique.

The low frequency circular fringes represent the parabolic distribution of the refractive index of the lens. As we mentioned previously, the high frequency speckle phase structures modulating the reconstructed wave front arising due to the used RPD plate. These high frequencies speckle noise can be eliminated using two different methods. The first method depends on the features of the RPD plate and because they are generally known the speckle noise can be numerically filtered. The second method is based on capturing two sets of intensity measurements. The first set takes place when the diffused light field illuminates the test object. The second set of intensity measurements is recorded after removing the object. Subtracting the phase distributions retrieved from the two cases, the phase of the investigated object remains. In this work we use the filtering approach to reduce the speckle noise. The filtered version of the retrieved phase distribution of the investigated lens is shown by Figure 9(a).

To quantitatively assess the retrieved phase distribution, the refractive index profile of the test lens is numerically determined. It has been calculated using a Matlab script for a lens with a known focal length of $f=150 \mathrm{~mm}$ and is depicted in Figure 9(b). Comparing the refractive profile of the retrieved phase and that one calculated numerically, it is seen that the concentric circular fringes agree well. For further discussion, the corresponding phase difference is shown by Figure 9(c). The standard deviation has been determined to be $0.35 \mathrm{rad}$, which means that both distributions deviate by an average of approximately $5.5 \%$. It is noted that the large deviations are mainly found close to the regions where the in- tensity is minimum. However, the remarkable agreement of the experimental and the numerical results greatly verifies the potential of the presented method for phase retrieval from a set of intensity observations. Additionally, compared to existing phase retrieval techniques involving time-consuming mechanical adjustments, the duration of the measurement process is considerably reduced because of the rapid switching time of the SLM used.

\section{CONCLUSION}

We have presented an experimental technique for the recovery of smooth wave fronts by means of the SLM-based phase retrieval technique and a phase diffuser having specific features. The main task of the diffuse illumination is to introduce significant diversity in the intensity measurements which is lacking in the case of smooth test wave fronts. The lack of diversity in the intensity measurements causes the iterative phase retrieval technique to converge to a wrong solution. The use of the SLM in a $4 f$-setup, owing to its fully-automated control, enables accurate and fast speckle intensity recording. It has been proven that this combination works and yields a rapid approach for the investigation of smooth wave fronts with active compensation for misalignments in the setup. The application in the shape measurement of a thin lens demonstrates the effectiveness of the technique.

\section{ACKNOWLEDGEMENTS}

The authors thank R. B. Bergmann and C. von Kopylow for continuous support and comments. Furthermore, the authors gratefully acknowledge the financial support of the Deutsche Forschungsgemeinschaft (DFG) for funding this work within the frame of project "DynaLiFeS" (BE1924/2-1) and the Collaborative Research Center SFB 747-B5. P. Almoro acknowledges the SFB 747-B5 (BIAS) and the University of the Philippines for his research visit and the National Research Council of the Philippines for the financial support.

\section{References}

[1] R. A. Consalves, "Phase retrieval and diversity in adaptive optics," Opt. Eng. 21, 829-832 (1982).

[2] R. W. Gerchberg, and W. 0. Saxton, "A practical algorithm for the determination of phase from image and diffraction plane pictures," Optik (Jena) 35, 237-246 (1972).

[3] R. Rolleston, and N. George, "Image reconstruction from partial Fresnel zone information," Appl. Opt. 25, 178-183 (1986).

[4] P. Bao, F. Zhang, G. Pedrini, and W. Osten, "Phase retrieval using multiple illumination wavelengths," Opt. Lett. 33, 309-311 (2008).

[5] M. Guizar-Sicairos, and J. R. Fienup, "Measurement of coherent $x$ ray focused beams by phase retrieval with transverse translation diversity," Opt. Express 17, 2670-2685, (2009).

[6] A. M. Maiden, M. J. Humphry, F. Zhang, and J. M. Rodenburg, "Superresolution imaging via ptychography," J. Opt. Soc. Am. A 28, 604-612 (2011). 
[7] P. Almoro, G. Pedrini, and W. Osten, “Complete wavefront reconstruction using sequential intensity measurements of a volume speckle field," Appl. Opt. 45, 8596-8605 (2006).

[8] J. Goodman, Introduction to Fourier Optics (2nd ed., McGraw-Hill, 1996).

[9] P. F. Almoro, and S. G. Hanson, "Object wave reconstruction by speckle illumination and phase retrieval," J. Europ. Opt. Soc. Rap. Public. 4, 09002 (2009).

[10] A. M. S. Maallo, P. F. Almoro, and S. G. Hanson, "Quantization analysis of speckle intensity measurements for phase retrieval," Appl. Opt. 49, 5087-5094 (2010).

[11] P. F. Almoro, G. Pedrini, P. N. Gundu, W. Osten, and S. G. Hanson, "Enhanced Wavefront Reconstruction by Random Phase Modulation with a Phase Diffuser," Opt. Lasers Eng. 49, 252-257 (2011).

[12] C. Falldorf, M. Agour, C. von Kopylow, and R. B. Bergmann, “Phase retrieval by means of a spatial light modulator in the Fourier domain of an imaging system," Appl. 0pt. 49, 1826-1830 (2010).

[13] M. Agour, C. Falldorf, C. von Kopylow, and R. B. Bergmann, "Automated compensation of misalignment in phase retrieval based on a spatial light modulator," Appl. 0pt. 50, 4779-4787 (2011).

[14] C. Falldorf, M. Agour, C. von Kopylow, and R. B. Bergmann, Proc. International Symposium on Optomechatronic Technologies (ISOT 2010), F. Janabi-Sharifi, and J. Kofman, eds., 1-5 (IEEE, 2010).

[15] C. Falldorf, M. Agour, C. von Kopylow, and R. B. Bergmann, "Phase Retrieval for Optical Inspection of Technical Components," J. Opt. 14, 065701 (2012).
[16] M. Agour, C. Falldorf, C. von Kopylow, and R. B. Bergmann, "The effect of misalignment in phase retrieval based on a spatial light modulator," Proc. SPIE 8082, 80820M (2011).

[17] Holoeye Photonics AG, PLUT0-07 in. HDTV LCOS Phase Only Kit Specification Sheet.

[18] M. Agour, C. Falldorf, E. Kolenovic, and C. von Kopylow, "Suppression of higher diffraction orders and intensity improvement of optically reconstructed holograms from a spatial light modulator," J. Opt. A: Pure Appl. Opt. 11, 105405 (2009).

[19] M. Agour, C. Falldorf, and C. von Kopylow, "Digital pre-filtering approach to improve optically reconstructed wavefields in optoelectronic holography," J. Opt. 12, 055401 (2010).

[20] C. Falldorf, M. Agour, C. von Kopylow, and R. B. Bergmann, "Design of an optical system for phase retrieval based on a spatial light modulator," AIP Conf. Proc. 1236, 259-264 (2010).

[21] P. F. Almoro, and S. G. Hanson, "Random phase plate for wavefront sensing via phase retrieval and a volume speckle field," Appl. Opt. 47, 2979-2987 (2008)

[22] J. Goodman, "Statistical properties of laser speckle patterns." in Laser Speckle and Related Phenomena, vol. 9 of Topics in Applied Physics, J. C. Dainty, ed, 9-75. (Springer, Berlin / Heidelberg, 1975)

[23] E. Kolenovic, W. Osten, and W. Jüptner, "Non-linear speckle phase changes in the image plane caused by out of plane displacement," Opt. Commun. 171, 333-344 (1999). 